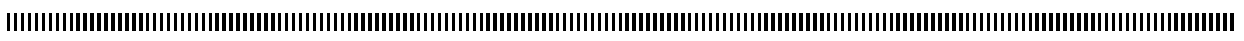
| |

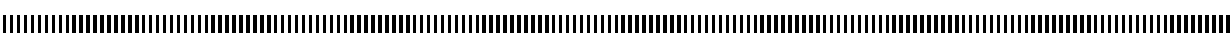

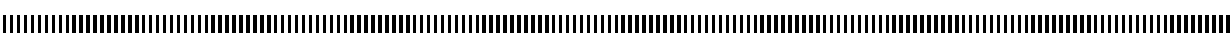

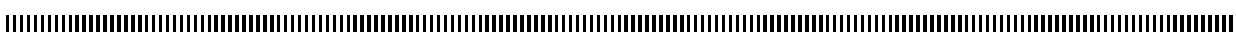

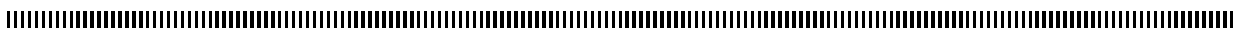

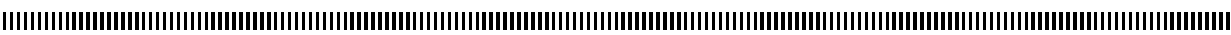

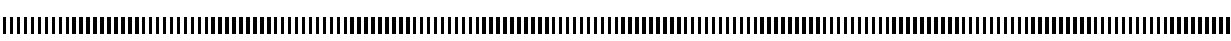

\title{
Shape optimization for the Stokes equations using topological sensitivity analysis
}

\author{
Hassine Maatoug \\ ENIT-LAMSIN \& FSM \\ Université Tunis El-Manar \\ Campus Universitaire, Le Belvédaire B.P. 37, 1002 Tunis \\ Tunisie \\ Maatoug.Hassine@enit.rnu.tn
}

|

RÉSUMÉ. Dans ce papier, on considère un problème d'optimisation de forme lié aux équations de Stokes. On propose une approche basée sur une analyse de sensibilité topologique. On donne un développement asymptotique d'une fonction coût par rapport à la perturbation du domaine par l'insertion d'un petit obstacle. Des résultats théoriques sont donnés en $2 \mathrm{D}$ et $3 \mathrm{D}$. Dans la partie numérique, on utilise cette approche pour optimiser la forme des tubes liant l'entrée aux sorties d'un cavité.

ABSTRACT. In this paper, we consider a shape optimization problem related to the Stokes equations. The proposed approach is based on a topological sensitivity analysis. It consists in an asymptotic expansion of a cost function with respect to the insertion of a small obstacle in the domain. The theoretical part of this work is discussed in both two and three dimensional cases. In the numerical part, we use this approach to optimize the shape of the tubes that connect the inlet to the outlets of the cavity maximizing the outflow rate.

MOTS-CLÉS : Optimisation de forme, optimisation topologique, gradient topologique, équations de Stokes.

KEYWORDS : Shape optimization, topological optimization, topological gradient, Stokes equations.

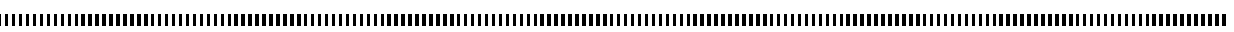




\section{Introduction}

Topological optimization is concerned with the variation of a cost function with respect to a topological modification of a domain. The most simple way of modifying the topology consists in creating a small hole in the domain. In the case of structural shape optimization, creating a hole means simply removing some material. In the case of fluid dynamics where the domain represents the fluid, creating a hole means inserting a small obstacle. The situation is similar in electromagnetism. Unlike the classical shape optimization, the topology of the domain may change during the optimization process. The objective is to find an optimal shape without any a priori assumption about its topology. The given approach is based on the analysis of the topological sensitivity. It provides an asymptotic expansion of a cost function with respect to a small topological perturbation of the domain. To present the basic idea, we consider $\Omega$ a domain of $\mathbb{R}^{d}, d=2,3$ and $j(\Omega)=J\left(\Omega, u_{\Omega}\right)$ a cost function to be minimized, where $u_{\Omega}$ is the solution to a given PDE problem defined in $\Omega$. For $\varepsilon>0$, let $\Omega_{\varepsilon}=\Omega \backslash \overline{\left(x_{0}+\varepsilon \omega\right)}$ be the domain obtained by removing a small part $\overline{\left(x_{0}+\varepsilon \omega\right)}$ from $\Omega$, where $x_{0} \in \Omega$ and $\omega \subset \mathbb{R}^{d}$ is a fixed bounded domain containing the origin. Then, an asymptotic expansion of the function $j$ is obtained in the following form :

$$
\begin{aligned}
& j\left(\Omega_{\varepsilon}\right)=j(\Omega)+f(\varepsilon) g\left(x_{0}\right)+o(f(\varepsilon)) \\
& f(\varepsilon)>0 \quad \forall \varepsilon>0, \quad \lim _{\varepsilon \rightarrow 0} f(\varepsilon)=0 .
\end{aligned}
$$

The function $g$ is called the topological sensitivity or topological gradient. It can be used as a descent direction of the domain optimization process. Obviously, if we want to minimize $j$, the "best" place to create an infinitesimal hole is there where $g(x)$ is the most negative. Starting with this observation, topological optimization algorithms can then be constructed [7].

The topological sensitivity analysis was introduced by Schumacher [17], Sokolowski and Zochowski [18] for the minimization of the compliance in linear elasticity with Neumann condition on the boundary of the inserted hole. A topological sensitivity framework using an adaptation of the adjoint method $[6,14]$ and a truncation technique was introduced by Masmoudi [14]. It was generalized in [9] to the elasticity equations in the case of arbitrary shaped holes. Recently, the topological asymptotic expansion was obtained for various problems[11, 12, 13, 3, 2].

In this paper we consider the Stokes problem. This problem is considered by Guillaume and Sid Idriss in [12]. They have obtained a topological asymptotic expansion for a large class of cost functions and arbitrary shaped holes. The approach used in [12] is based on an adaptation of the adjoint method and a domain truncation technique that provides an equivalent formulation of the PDE in a fixed functional space. In this work we present a simplified topological sensitivity analysis for the Stokes equations without using the truncation technique. Our tools is an adjoint method that takes into account the variation of the functional space when a Dirichlet condition is applied on the moving part of the domain (Paragraph 3.1). Such an adjoint method brings several technical simplifications. The asymptotic expansion we obtain (Theorems 3.2 and 3.3) is based on an asymptotic formula for the velocity in the perturbed domain $\Omega_{\varepsilon}$ (Proposition 3.1).

In the numerical part, we propose a shape optimization problem. We consider an incompressible fluid flow in a cavity $\Omega$ having one inlet $\Gamma_{\text {in }}$ and some outlets $\Gamma_{\text {out }}^{i}, i=1, m$. 


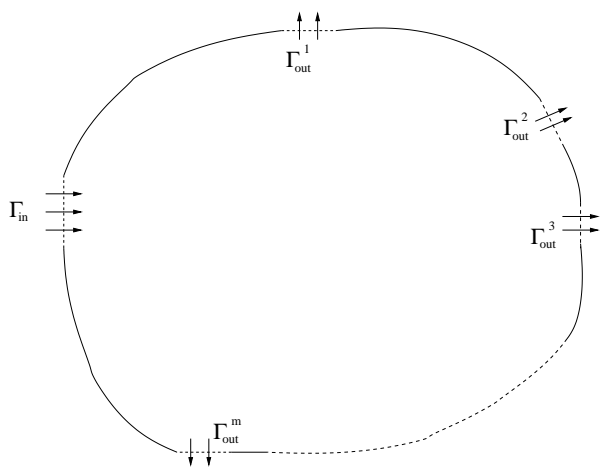

Figure 1. The cavity $\Omega$.

(see Figure 1). Our objective is to determine the optimal shape of the tubes that connect the inlet to the outlets of the cavity maximizing the outflow rate by inserting some small obstacles in the cavity.

The paper is organized as follows. In section 2, we give a precise statement of the optimization problem. In section 3, we describe the adjoint method and we present the main results. Finally in Section 4, we present some numerical experiments.

\section{Formulation of the problem}

Consider a viscous incompressible fluid $\mathcal{F}$, governed by Stokes equations, in steadystate regime. The Eulerian velocity vector $u$ and the pressure $p$ of $\mathcal{F}$ fulfil the system

$$
\left\{\begin{aligned}
-\nu \Delta u+\nabla p & =F & & \text { in } \Omega \\
\operatorname{div} u & =0 & & \text { in } \Omega \\
u & =0 & & \text { on } \Gamma,
\end{aligned}\right.
$$

where $\Omega \subset \mathbb{R}^{d}, d=2,3$, is the domain occupied by the fluid, $\nu$ is the (constant) kinematic viscosity coefficient of $\mathcal{F}$, and $F$ is a given body force per unit of mass.

\subsection{Stokes equations in the perturbed domain}

We denote by $\Omega_{\varepsilon}$ the perturbed domain, obtained from inserting a small obstacle $\omega_{\varepsilon}$ in $\Omega$. We suppose that the obstacle has the form $\omega_{\varepsilon}=x_{0}+\varepsilon \omega$, where $x_{0} \in \Omega, \varepsilon>0$ and $\omega$ is a given fixed and bounded domain of $\mathbb{R}^{d}$, containing the origin, whose boundary $\partial \omega$ is connected and piecewise of class $\mathcal{C}^{1}$.

In $\Omega_{\varepsilon}$, the velocity $u_{\varepsilon}$ and the pressure $p_{\varepsilon}$ of $\mathcal{F}$ are solution to

$$
\left\{\begin{aligned}
-\nu \Delta u_{\varepsilon}+\nabla p_{\varepsilon} & =F & & \text { in } \Omega_{\varepsilon} \\
\operatorname{div} u_{\varepsilon} & =0 & & \text { in } \Omega_{\varepsilon} \\
u_{\varepsilon} & =0 & & \text { on } \Gamma \\
u_{\varepsilon} & =0 & & \text { on } \partial \omega_{\varepsilon} .
\end{aligned}\right.
$$

Note that for $\varepsilon=0, \Omega_{0}=\Omega$ and $\left(u_{0}, p_{0}\right)$ is solution to

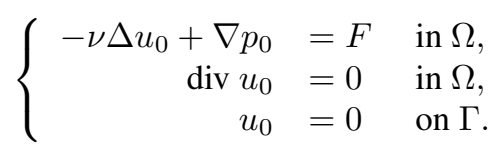




\subsection{Topological optimization problem}

Consider now a cost function $j(\varepsilon)$ of the form

$$
j(\varepsilon)=J_{\varepsilon}\left(\Omega_{\varepsilon}, u_{\varepsilon}\right),
$$

where $J_{\varepsilon}$ is defined on $H^{1}\left(\Omega_{\varepsilon}\right)^{d}$ for $\varepsilon \geq 0$ and $J_{0}$ is differentiable with respect to $u$, its derivative being denoted by $D J_{0}(u)$.

Our aim is to determine the optimal location of the obstacle $\omega_{\varepsilon}$ in the domain $\Omega$ in order to minimize the cost function $J_{\varepsilon}\left(\Omega_{\varepsilon}, u_{\varepsilon}\right)$. Then, the optimization problem we consider is given as follows :

$$
\begin{aligned}
& \min _{\omega_{\varepsilon} \subset \Omega} J_{\varepsilon}\left(\Omega_{\varepsilon}, u_{\varepsilon}\right) \text { such that, for some } p_{\varepsilon}, \\
& \left(u_{\varepsilon}, p_{\varepsilon}\right) \text { is a solution of (1) in } \Omega_{\varepsilon} .
\end{aligned}
$$

To this end, we will derive a topological asymptotic expansion of the function $j$ with respect to $\varepsilon$.

\section{Topological sensitivity analysis}

In this section we consider a topological sensitivity analysis for the Stokes equations. We present a topological asymptotic expansion of a cost function $j$ with respect to the insertion of a small obstacle $\omega_{\varepsilon}$ inside the domain $\Omega$. The proposed approach is based on the following general adjoint method.

\subsection{General adjoint method}

Let $\left(\mathcal{V}_{\varepsilon}\right)_{\varepsilon \geq 0}$ be a family of Hilbert spaces depending on the parameter $\varepsilon$, such that, $\forall \varepsilon \geq 0 \mathcal{V}_{\varepsilon} \hookrightarrow \mathcal{V}_{0}$. For $\varepsilon \geq 0$, we consider

- $\mathcal{A}_{\varepsilon}: \mathcal{V}_{\varepsilon} \times \mathcal{V}_{\varepsilon} \longrightarrow \mathbb{R}$ a bilinear, continuous and coercive form on $\mathcal{V}_{\varepsilon}$,

- $l_{\varepsilon}: \mathcal{V}_{\varepsilon} \longrightarrow \mathbb{R}$ a linear and continuous form on $\mathcal{V}_{\varepsilon}$.

We assume that for all $\varepsilon \geq 0$, the problem

$$
\mathcal{A}_{\varepsilon}\left(u_{\varepsilon}, w\right)=l_{\varepsilon}(w), \quad \forall w \in \mathcal{V}_{\varepsilon}
$$

has a unique solution $u_{\varepsilon} \in \mathcal{V}_{\varepsilon}$.

Consider now a cost function of the form $j(\varepsilon)=J_{\varepsilon}\left(u_{\varepsilon}\right)$, where $J_{\varepsilon}$ is defined on $\mathcal{V}_{\varepsilon}$ for $\varepsilon \geq 0$ and $J_{0}$ is differentiable with respect to $u$, its derivative being denoted by $D J_{0}(u)$.

Our aim is to derive an asymptotic expansion of $j$ with respect to $\varepsilon$. We consider the following assumptions.

Hypothesis 3.1 There exist a real number $\delta \mathcal{A}$ and a scalar function $f: \mathbb{R}_{+} \longrightarrow \mathbb{R}_{+}$ such that $\forall \varepsilon \geq 0$

$$
\begin{aligned}
& \mathcal{A}_{0}\left(u_{0}-u_{\varepsilon}, v_{0}\right)=f(\varepsilon) \delta \mathcal{A}+o(f(\varepsilon)), \\
& \lim _{\varepsilon \rightarrow 0} f(\varepsilon)=0
\end{aligned}
$$

ARIMA - numéro spécial TAM TAM'05 
where $v_{0} \in \mathcal{V}_{0}$ is the solution to the adjoint problem

$$
\mathcal{A}_{0}\left(w, v_{0}\right)=-D J_{0}\left(u_{0}\right) w, \quad \forall w \in \mathcal{V}_{0} .
$$

Hypothesis 3.2 There exists a real number $\delta J$ such that $\forall \varepsilon \geq 0$

$$
J_{\varepsilon}\left(u_{\varepsilon}\right)-J_{0}\left(u_{0}\right)=D J_{0}\left(u_{0}\right)\left(u_{\varepsilon}-u_{0}\right)+f(\varepsilon) \delta J+o(f(\varepsilon)) .
$$

Under the assumptions 3.1 and 3.2, we have the following theorem.

Theorem 3.1 If the assumptions 3.1 and 3.2 hold, the function $j$ has the following asymptotic expansion

$$
j(\varepsilon)=j(0)+f(\varepsilon)(\delta \mathcal{A}+\delta J)+o(f(\varepsilon)) .
$$

Proof : From hypothesis 3.2, we have

$$
\begin{aligned}
j(\varepsilon)-j(0) & =J_{\varepsilon}\left(u_{\varepsilon}\right)-J_{0}\left(u_{0}\right), \\
& =D J_{0}\left(u_{0}\right)\left(u_{\varepsilon}-u_{0}\right)+f(\varepsilon) \delta J+o(f(\varepsilon)) .
\end{aligned}
$$

Using (6) and hypothesis 3.1

$$
\begin{aligned}
j(\varepsilon)-j(0) & =\mathcal{A}_{0}\left(u_{0}-u_{\varepsilon}, v_{0}\right)+f(\varepsilon) \delta J+o(f(\varepsilon)) \\
& =f(\varepsilon)(\delta \mathcal{A}+\delta J)+o(f(\varepsilon)) .
\end{aligned}
$$

\subsection{Topological sensitivity for the Stokes problem}

In this section, we derive a topological asymptotic expansion for the Stokes equations. In order to apply the adjoint method described in the previous paragraph, first we establish a variational problem associated to the Stokes system. From the weak variational formulation of (4), we deduce that $u_{\varepsilon} \in \mathcal{V}_{\varepsilon}$ is solution to

$$
\mathcal{A}_{\varepsilon}\left(u_{\varepsilon}, w\right)=l_{\varepsilon}(w), \quad \forall w \in \mathcal{V}_{\varepsilon}
$$

where the functional space $\mathcal{V}_{\varepsilon}$, the bilinear form $\mathcal{A}_{\varepsilon}$ and the linear form $l_{\varepsilon}$ are defined by

$$
\begin{aligned}
& \mathcal{V}_{\varepsilon}=\left\{w \in H_{0}^{1}\left(\Omega_{\varepsilon}\right), \operatorname{div} w=0 \text { in } \Omega_{\varepsilon}\right\} \\
& \mathcal{A}_{\varepsilon}(v, w)=\nu \int_{\Omega_{\varepsilon}} \nabla v \nabla w \mathrm{dx}, \quad \forall u, v \in \mathcal{V}_{\varepsilon} \\
& l_{\varepsilon}(w)=\int_{\Omega_{\varepsilon}} F w \mathrm{dx}, \quad \forall w \in \mathcal{V}_{\varepsilon} .
\end{aligned}
$$

Next we have to distinguish the cases $d=2$ and $d=3$. This is due to the fact that the fundamental solutions to the Stokes equations in $\mathbb{R}^{2}$ and $\mathbb{R}^{3}$ have an essentially different asymptotic behaviour at infinity. 


\subsubsection{The three dimensional case}

Let $(U, P)$ denote a solution to

$$
\left\{\begin{aligned}
-\nu \Delta U+\nabla P & =0 & & \text { in } \mathbb{R}^{3} \backslash \bar{\omega} \\
\operatorname{div} U & =0 & & \text { in } \mathbb{R}^{3} \backslash \bar{\omega} \\
U & \longrightarrow 0 & & \text { at } \infty \\
U & =-u_{0}\left(x_{0}\right) & & \text { on } \partial \omega .
\end{aligned}\right.
$$

The existence of $(U, P)$ is most easily established by representing it as a single layer potential on $\partial \omega$ (see [8])

$$
\begin{array}{ll}
U(y)=\int_{\partial \omega} E(y-x) \eta(x) \mathrm{ds}(x), & y \in \mathbb{R}^{3} \backslash \bar{\omega} \\
P(y)=\int_{\partial \omega} \Pi(y-x) \eta(x) \mathrm{ds}(x), & y \in \mathbb{R}^{3} \backslash \bar{\omega}
\end{array}
$$

where $(E, \Pi)$ is the fundamental solution of the Stokes equations

$$
E(y)=\frac{1}{8 \pi \nu r}\left(I+e_{r} e_{r}^{T}\right), \quad \Pi(y)=\frac{y}{4 \pi r^{3}},
$$

with $r=\|y\|, e_{r}=y / r$ and $e_{r}^{T}$ is the transposed vector of $e_{r}$. The functions $\eta \in$ $H^{-1 / 2}(\partial \omega)^{3}$ is the solution to the boundary integral equation,

$$
\int_{\partial \omega} E(y-x) \eta(x) \mathrm{ds}(x)=-u_{0}\left(x_{0}\right), \quad \forall y \in \partial \omega .
$$

One can observe that the function $\eta$ is determined up to a function proportional to the normal, hence it is unique in $H^{-1 / 2}(\partial \omega)^{3} / \mathbb{R} n$.

We start the derivation of the topological asymptotic expansion with the following estimate of the $H^{1}\left(\Omega_{\varepsilon}\right)$ norm of $u_{\varepsilon}(x)-u_{0}(x)-U(x / \varepsilon)$. This estimate plays a crucial role in the derivation of our topological asymptotic expansion. It describes the velocity perturbation caused by the presence of the small obstacle $\omega_{\varepsilon}$.

Proposition 3.1 There exists $c>0$, independent on $\varepsilon$, such that for all $\varepsilon>0$ we have

$$
\left\|u_{\varepsilon}(x)-u_{0}(x)-U(x / \varepsilon)\right\|_{1, \Omega_{\varepsilon}} \leq c \varepsilon
$$

The following corollary follows from Proposition 3.1. It gives the behaviour of the velocity $u_{\varepsilon}$ when inserting an obstacle. The principal term of this perturbation is given by the function $U$, solution to (10).

Corollary 3.1 We have

$$
u_{\varepsilon}(x)=u_{0}(x)+U(x / \varepsilon)+O(\varepsilon), \quad x \in \Omega_{\varepsilon} .
$$

We are now ready to derive the topological asymptotic expansion of the cost function $j$. It consists in computing the variation $j(\varepsilon)-j(0)$ when inserting a small obstacle inside the domain. The leading term of this variation involves the function $\eta$, the solution to the boundary integral equation (11). The main result is described by Theorem 3.2.

ARIMA - numéro spécial TAM TAM'05

ARIMA 
Theorem 3.2 If Hypothesis 3.1 holds, the function $j$ has the following asymptotic expansion

$$
j(\varepsilon)=j(0)+\varepsilon\left[\left(-\int_{\partial \omega} \eta(y) d s(y)\right) \cdot v_{0}\left(x_{0}\right)+\delta J\right]+o(\varepsilon) .
$$

In the particular case where $\omega=B(0,1)$, the density $\eta$ is given explicitly $\eta(y)=$ $-\frac{3 \nu}{2} u_{0}\left(x_{0}\right), \quad \forall y \in \partial \omega$.

Corollary 3.2 If $\omega=B(0,1)$, under the hypotheses of theorem 3.2, we have

$$
j(\varepsilon)=j(0)+\varepsilon\left[6 \pi \nu u_{0}\left(x_{0}\right) \cdot v_{0}\left(x_{0}\right)+\delta J\right]+o(\varepsilon) .
$$

\subsubsection{The two dimensional case}

In this paragraph, we present the topological asymptotic expansion for the Stokes equations in the two dimensional case. The result is obtained using the same technique described in the previous paragraph. The unique difference comes from the expression of the fundamental solution of the Stokes equations. In this case $(E, \Pi)$ is given by

$$
E(y)=\frac{1}{4 \pi \nu}\left(-\log (r) I+e_{r} e_{r}^{T}\right), \quad \Pi(y)=\frac{y}{2 \pi r^{2}} .
$$

Theorem 3.3 Under the same hypotheses of theorem 3.2, the function $j$ has the following asymptotic expansion

$$
j(\varepsilon)=j(0)+\frac{-1}{\log (\varepsilon)}\left[4 \pi \nu u_{0}\left(x_{0}\right) \cdot v_{0}\left(x_{0}\right)+\delta J\right]+o\left(\frac{-1}{\log (\varepsilon)}\right) .
$$

\subsection{Proof}

First we need some definitions and preliminary lemmas.

Let $\mathcal{O}$ be a bounded open domain of $\mathbb{R}^{3}$ and $\partial \mathcal{O}$ its boundary, assumed polygonal and simply connected.

- We denote by $H_{V}^{1 / 2}(\partial \mathcal{O})$ the subspace of traces $\left(H_{V}^{1 / 2}(\partial \mathcal{O}) \subset H^{1 / 2}(\partial \mathcal{O})\right)$

$$
H_{V}^{1 / 2}(\partial \mathcal{O})=\left\{\phi \in H^{1 / 2}(\partial \mathcal{O}), \quad \int_{\partial \mathcal{O}} \phi . n \mathrm{ds}=0\right\} .
$$

- Let $\varepsilon>0$; for a function $u$ defined on a given $\mathcal{O}$, we define the function $\tilde{u}$ on $\tilde{\mathcal{O}}:=\mathcal{O} / \varepsilon$ by

$$
\tilde{u}(y)=u(x), \quad y=x / \varepsilon .
$$

We have the following relations

$$
|u|_{1, \mathcal{O}}=\varepsilon^{1 / 2}|\tilde{u}|_{1, \tilde{\mathcal{O}}},\|u\|_{0, \mathcal{O}}=\varepsilon^{3 / 2}\|\tilde{u}\|_{0, \tilde{\mathcal{O}}}
$$

- Let $R>0$ be such that the closed ball $\overline{B\left(x_{0}, R\right)}$ is included in $\Omega$ and $\bar{\omega}_{\varepsilon} \subset B\left(x_{0}, R\right)$. We denote by $\Gamma_{R}$ the boundary of $B\left(x_{0}, R\right)$ and we define the domains

$$
\Omega_{R}=\Omega \backslash \overline{B\left(x_{0}, R\right)} \text { and } D_{\varepsilon}=B\left(x_{0}, R\right) \backslash \overline{\omega_{\varepsilon}} .
$$




\subsubsection{Preliminary lemmas}

The aim of this section is to give some technical results which will be used in section 3.3.2 and 3.3.3. For more details and proof one may consult [12] or [13].

Lemma 3.1 For $\phi \in H_{V}^{1 / 2}(\partial \omega)^{3}$; let $(w, s)$ be the solution to the Stokes exterior problem

$$
\left\{\begin{aligned}
-\nu \Delta w+\nabla s & =0 & & \text { in } \mathbb{R}^{3} \backslash \bar{\omega} \\
\operatorname{div} w & =0 & & \text { in } \mathbb{R}^{3} \backslash \bar{\omega} \\
w & =0 & & \text { at infinity } \\
w & =\phi & & \text { on } \partial \omega
\end{aligned}\right.
$$

Then, there exist a constants $c>0$, independent of $\phi$ and $\varepsilon$, such that

$$
\begin{aligned}
\|w\|_{0, D_{\varepsilon} / \varepsilon} & \leq c \varepsilon^{-1 / 2}\|\phi\|_{1 / 2, \partial \omega} \\
\|w\|_{0, \Omega_{R} / \varepsilon} & \leq c \varepsilon^{-1 / 2}\|\phi\|_{1 / 2, \partial \omega} \\
|w|_{1, D_{\varepsilon} / \varepsilon} & \leq c\|\phi\|_{1 / 2, \partial \omega} \\
|w|_{1, \Omega_{R} / \varepsilon} & \leq c \varepsilon^{1 / 2}\|\phi\|_{1 / 2, \partial \omega} .
\end{aligned}
$$

Lemma 3.2 For a given $\varepsilon>0, \varphi \in H_{V}^{1 / 2}(\Gamma)^{3}$ and $\psi \in H^{1}\left(D_{0}\right)^{3}$ such that div $\psi=0$, let $\left(v_{\varepsilon}, q_{\varepsilon}\right)$ be the solution to the Stokes problem

$$
\left\{\begin{aligned}
-\nu \Delta v_{\varepsilon}+\nabla q_{\varepsilon} & =0 & & \text { in } \Omega_{\varepsilon} \\
\operatorname{div} v_{\varepsilon} & =0 & & \text { in } \Omega_{\varepsilon} \\
v_{\varepsilon} & =\varphi & & \text { on } \Gamma \\
v_{\varepsilon} & =\psi & & \text { on } \partial \omega_{\varepsilon}
\end{aligned}\right.
$$

Then, there exist a constant $c>0$ (independent of $\varphi, \psi$ and $\varepsilon$ ), and $\varepsilon_{1}>0$ such that for all $0<\varepsilon<\varepsilon_{1}$

$$
\begin{aligned}
\left|v_{\varepsilon}\right|_{1, \Omega_{R}} & \leq c\left(\|\varphi\|_{1 / 2, \Gamma}+\varepsilon\|\psi(\varepsilon y)\|_{1 / 2, \partial \omega}\right) \\
\left\|v_{\varepsilon}\right\|_{0, D_{\varepsilon}} & \leq c\left(\|\varphi\|_{1 / 2, \Gamma}+\varepsilon\|\psi(\varepsilon y)\|_{1 / 2, \partial \omega}\right) \\
\left|v_{\varepsilon}\right|_{1, D_{\varepsilon}} & \leq c\left(\|\varphi\|_{1 / 2, \Gamma}+\varepsilon^{1 / 2}\|\psi(\varepsilon y)\|_{1 / 2, \partial \omega}\right) .
\end{aligned}
$$

\subsubsection{Proof of Proposition 3.1}

Define

$$
z_{\varepsilon}(x)=u_{\varepsilon}(x)-u_{0}(x)-U(x / \varepsilon), \quad s_{\varepsilon}(x)=p_{\varepsilon}(x)-p_{0}(x)-P(x / \varepsilon) .
$$

From (1), (2) and (10) it follows that $\left(z_{\varepsilon}, s_{\varepsilon}\right)$ is solution to

$$
\left\{\begin{aligned}
-\nu \Delta z_{\varepsilon}+\nabla s_{\varepsilon} & =0 & & \text { in } \Omega_{\varepsilon} \\
\operatorname{div} z_{\varepsilon} & =0 & & \text { in } \Omega_{\varepsilon} \\
z_{\varepsilon} & =-U(x / \varepsilon) & & \text { on } \Gamma \\
z_{\varepsilon} & =-u_{0}(x)+u_{0}\left(x_{0}\right) & & \text { on } \partial \omega_{\varepsilon} .
\end{aligned}\right.
$$

Due to Lemma 3.2, there exists a constant $c>0$, independent of $\varepsilon$, such that

$$
\left\|z_{\varepsilon}\right\|_{1, \Omega_{\varepsilon}} \leq c\left(\|U(x / \varepsilon)\|_{1 / 2, \Gamma}+\varepsilon^{1 / 2}\left\|u_{0}\left(x_{0}+\varepsilon x\right)-u_{0}\left(x_{0}\right)\right\|_{1 / 2, \partial \omega}\right) .
$$


By trace theorem

$$
\|U(x / \varepsilon)\|_{1 / 2, \Gamma} \leq\|U(x / \varepsilon)\|_{0, \Omega_{R}}+|U(x / \varepsilon)|_{1, \Omega_{R}} .
$$

Changing variables and using (12)

$$
\|U(x / \varepsilon)\|_{1 / 2, \Gamma} \leq \varepsilon^{3 / 2}\|U(x / \varepsilon)\|_{0, \Omega_{R} / \varepsilon}+\varepsilon^{1 / 2}|U(x / \varepsilon)|_{1, \Omega_{R} / \varepsilon} .
$$

Due to Lemma 3.1 we obtain

$$
\|U(x / \varepsilon)\|_{1 / 2, \Gamma} \leq c \varepsilon .
$$

Expanding $u_{0}\left(x_{0}+\varepsilon x\right)=u_{0}\left(x_{0}\right)+\varepsilon \nabla u_{0}\left(\xi_{x}\right) x, \xi_{x} \in \omega_{\varepsilon}$, and using the fact that $\nabla u_{0}$ is uniformly bounded, the second term in (14) may be estimated by

$$
\left\|u_{0}\left(x_{0}+\varepsilon x\right)-u_{0}\left(x_{0}\right)\right\|_{1 / 2, \partial \omega} \leq c \varepsilon .
$$

Finally, combining (14), (15) and (16) we deduce the desired estimate

$$
\left\|u_{\varepsilon}(x)-u_{0}(x)-U(x / \varepsilon)\right\|_{1, \Omega_{\varepsilon}} \leq c \varepsilon .
$$

\subsubsection{Proof of Theorem 3.2}

Due to Theorem 3.1, it suffices to derive an asymptotic expansion of $\mathcal{A}_{0}\left(u_{\varepsilon}-u_{0}, v_{0}\right)$. Using (8) and the fact that $u_{\varepsilon}=0$ in $\omega_{\varepsilon}$, we have

$$
\begin{aligned}
\mathcal{A}_{0}\left(u_{\varepsilon}-u_{0}, v_{0}\right) & =\nu \int_{\Omega} \nabla\left(u_{\varepsilon}-u_{0}\right) \nabla v_{0} \mathrm{dx} \\
& =-\nu \int_{\omega_{\varepsilon}} \nabla u_{0} \nabla v_{0} \mathrm{dx}+\nu \int_{\Omega_{\varepsilon}} \nabla\left(u_{\varepsilon}-u_{0}\right) \nabla v_{0} \mathrm{dx} .
\end{aligned}
$$

Thanks to elliptic regularity, $\nabla u_{0}$ and $\nabla v_{0}$ are uniformly bounded on $\omega_{\varepsilon}$, then it follows that

$$
-\nu \int_{\omega_{\varepsilon}} \nabla u_{0} \nabla v_{0} \mathrm{dx}=O\left(\varepsilon^{3}\right) .
$$

Using (14), the second term in (17) can be written

$$
\nu \int_{\Omega_{\varepsilon}} \nabla\left(u_{\varepsilon}-u_{0}\right) \nabla v_{0} \mathrm{dx}=\nu \int_{\Omega_{\varepsilon}} \nabla U(x / \varepsilon) \nabla v_{0} \mathrm{dx}+\nu \int_{\Omega_{\varepsilon}} \nabla z_{\varepsilon} \nabla v_{0} \mathrm{dx} .
$$

Using Green formula and taking into account that $v_{0 \mid \Gamma}=0$, we obtain

$$
\nu \int_{\Omega_{\varepsilon}} \nabla\left(u_{\varepsilon}-u_{0}\right) \nabla v_{0} \mathrm{dx}=\int_{\partial \omega_{\varepsilon}} \sigma(U, P)(x / \varepsilon) \cdot \mathbf{n} v_{0} \mathrm{ds}+\int_{\partial \omega_{\varepsilon}} \sigma\left(z_{\varepsilon}, s_{\varepsilon}\right) \cdot \mathbf{n} v_{0} \mathrm{ds}
$$

where $\sigma$ is the stress tensor; $\sigma(U, P)=\nu\left(\nabla U+\nabla U^{T}\right)-P I$ and $\sigma\left(z_{\varepsilon}, s_{\varepsilon}\right)=\nu\left(\nabla z_{\varepsilon}+\right.$ $\left.\nabla z_{\varepsilon}^{T}\right)-s_{\varepsilon} I$.

By trace theorem, Proposition 3.1 and the fact that $v_{0}$ is uniformly bounded on $\omega_{\varepsilon}$

$$
\left|\int_{\partial \omega_{\varepsilon}} \sigma\left(z_{\varepsilon}, s_{\varepsilon}\right) \cdot \mathbf{n} v_{0} \mathrm{ds}\right| \leq\left\|z_{\varepsilon}\right\|_{1, \Omega_{\varepsilon}}\left\|v_{0}\right\|_{1, \omega_{\varepsilon}}=o(\varepsilon)
$$


Changing variables,

$$
\int_{\partial \omega_{\varepsilon}} \sigma(U, P)(x / \varepsilon) \cdot \mathbf{n} v_{0} \mathrm{ds}=\varepsilon \int_{\partial \omega} \sigma(U, P)(y) \cdot \mathbf{n} v_{0}\left(x_{0}+\varepsilon y\right) \mathrm{ds}(\mathrm{y}) .
$$

Expanding $v_{0}\left(x_{0}+\varepsilon y\right)=v_{0}\left(x_{0}\right)+\varepsilon \nabla v_{0}\left(\xi_{y}\right) y, \xi_{y} \in \omega_{\varepsilon}$, and using the fact that $\nabla v_{0}$ is uniformly bounded,

$$
\int_{\partial \omega_{\varepsilon}} \sigma(U, P)(y) \cdot \mathbf{n} v_{0}\left(x_{0}+\varepsilon y\right) \mathrm{ds}=\varepsilon\left(\int_{\partial \omega} \sigma(U, P)(y) \cdot \mathbf{n} \mathrm{ds}\right) v_{0}\left(x_{0}\right)+O\left(\varepsilon^{2}[20]\right.
$$

Due to the jump condition of the single layer potential (see [8]),

$$
\sigma(U, P)=-\eta+\sigma(W, Q)
$$

where $(W, Q)$ is the solution to the associated interior problem

$$
\left\{\begin{aligned}
-\nu \Delta W+\nabla Q & =0 & & \text { in } \omega \\
\operatorname{div} W & =0 & & \text { in } \omega \\
W & =U & & \text { on } \partial \omega .
\end{aligned}\right.
$$

From the fact that $\operatorname{div} \sigma(W, Q)=-\nu \Delta W+\nabla Q=0$ in $\omega$, we have

$$
\int_{\partial \omega} \sigma(W, Q)(y) \cdot \mathbf{n} \mathrm{ds}=0 .
$$

Therefore

$$
\int_{\partial \omega_{\varepsilon}} \sigma(U, P)(x / \varepsilon) \cdot \mathbf{n} v_{0} \mathrm{ds}=-\varepsilon\left(\int_{\partial \omega} \eta(y) \mathrm{ds}\right) \cdot v_{0}\left(x_{0}\right)+O\left(\varepsilon^{2}\right) .
$$

From equations (17), (18), (19), (20) and (21) we deduce

$$
\mathcal{A}_{0}\left(u_{\varepsilon}-u_{0}, v_{0}\right)=-\varepsilon\left(\int_{\partial \omega} \eta(y) \mathrm{ds}\right) \cdot v_{0}\left(x_{0}\right)+o(\varepsilon) .
$$

Then, Hypothesis 3.1 holds with

$$
\delta \mathcal{A}=-\int_{\partial \omega} \eta(y) \text { ds and } f(\varepsilon)=\varepsilon .
$$

\section{Numerical results}

Here, we limit ourselves to the two dimensional case. We consider a cavity with one inlet $\Gamma_{\text {in }}$ and three outlets $\Gamma_{\text {out }}^{i}, i=1,2,3$, having the same section. We assume that the flow satisfies the following boundary conditions :

- On the inlet $\Gamma_{i n}$ we define the normal component of the stress tensor:

$$
\sigma(u, p) . n=\phi \text { on } \Gamma_{i n},
$$

ARIMA - numéro spécial TAM TAM'05 
where $\sigma(u, p)=\nu\left(\nabla u+\nabla u^{T}\right)-p I$ and $I$ is the $2 \times 2$ identity matrix.

- On the outlets, we use a free surface boundary condition :

$$
\sigma(u, p) . n=0 \text { on } \cup_{i=1}^{3} \Gamma_{\text {out }}^{i} .
$$

The cost function measuring the outflow rate is given by

$$
J(u)=\sum_{i=1}^{3} \int_{\Gamma_{\text {out }}^{i}}|u \cdot \mathbf{n}| \mathrm{ds} .
$$

Our implementation is based on the following optimization algorithm. We apply an iterative process to build a sequence of geometries $\left(\Omega_{k}\right)_{k \geq 0}$ with $\Omega_{0}=\Omega$. At the k-th iteration, the topological gradient is denoted by $g_{k}$, and the new geometry $\Omega_{k+1}$ is defined by a level set curve of $g_{k}$.

\section{The algorithm :}

- Initialization : choose $\Omega_{0}=\Omega$, and set $k=0$.

- Repeat until $g_{k} \geq 0$ in $\Omega_{k}$ :

- solve the direct problem and its associated adjoint problem in $\Omega_{k}$,

- compute the topological sensitivity $g_{k}$,

- set $\Omega_{k+1}=\left\{x \in \Omega_{k}, \quad g_{k}(x) \geq c_{k}\right\}$, where $c_{k}$ is chosen in such a way that the cost function decreases as most as possible,

$-k \longleftarrow k+1$.

This algorithm can be seen as a descent method where the descent direction is determined by the topological sensitivity $g_{k}$ and the step length is given by the volume variation $\operatorname{meas}\left(\Omega_{k} \backslash \Omega_{k+1}\right)$.

In the above algorithm, the direct and the adjoint problems are discretized by a finite element method using a Reynold number $R e=100$. The computation of the approximate solution is achieved by Uzawa algorithm.

The results are presented in figures $\mathbf{2}$ and $\mathbf{3}$. We illustrate the topological gradient and the geometries obtained during the optimization process in figure $\mathbf{2}$. The topological gradient corresponding to the initial geometry is shown in Figure 2-(a). The shape obtained after optimization is presented in figure 2-(d). In figure 3, we present the velocity field computed in the geometry obtained during the optimization process. The optimal velocity is given in figure 3-(d).

\section{Bibliographie}

[1] G. Allaire, F. Jouve, A-M. Toader « Structural optimization using sensitivity analysis and a level-set method », J. Comput. Phys., n ${ }^{\circ} 194$ (1), 2004, 363-393.

[2] S. Amstutz « The topological asymptotic for the Helmholtz equation : insertion of a hole, a crack and a dielectric object », Rapport MIP, $\mathrm{n}^{\circ}$ 03-05, 2003.

[3] S. Amstutz, M. Masmoudi, B. Samet « The topological asymptotic for the Helmholtz equation », SIAM J. Control Optim. n ${ }^{\circ}$ ?, vol. ?. 


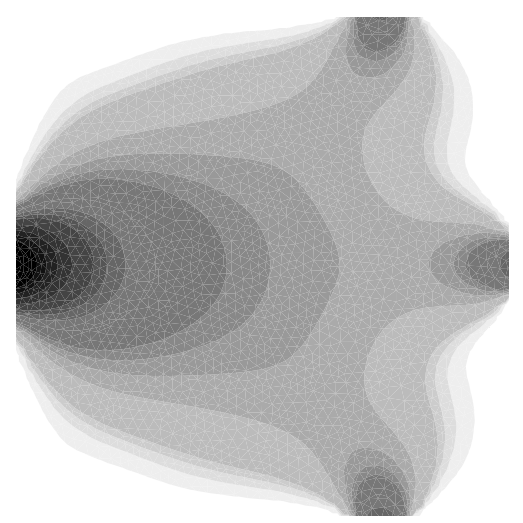

(a)

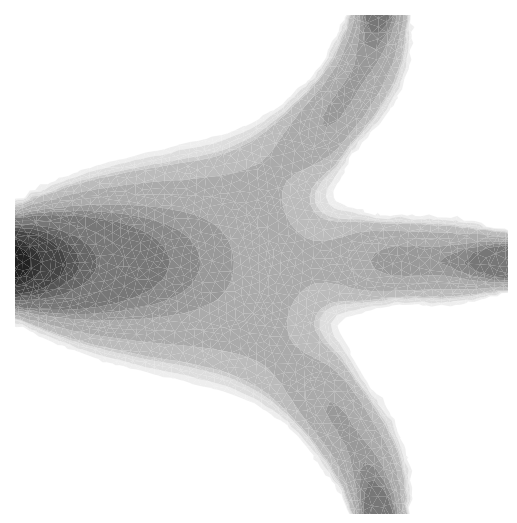

(c)

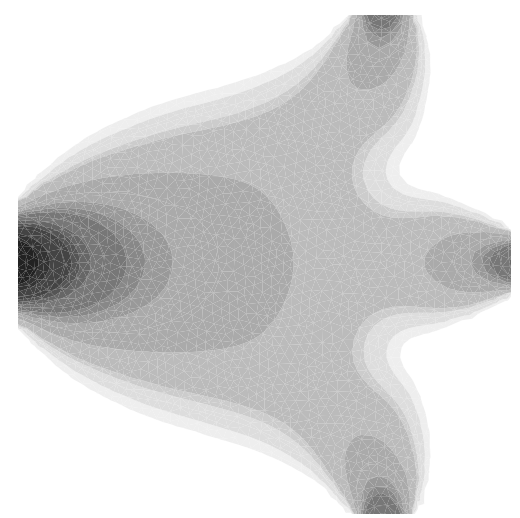

(b)

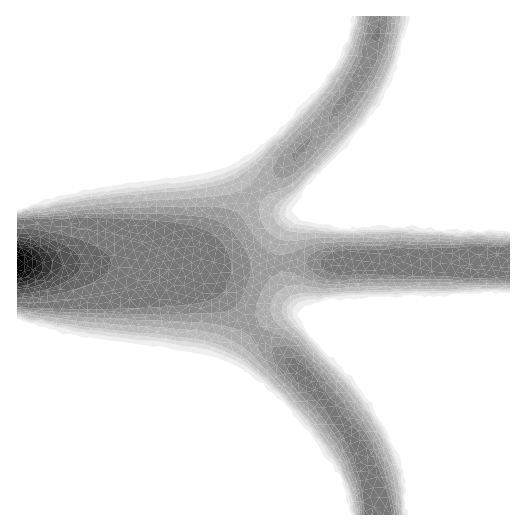

(d)

Figure 2. The topological gradient describing the geometry during optimization process.

[4] M. Bendsoe « Optimal topology design of continuum structure : an introduction », Technical report, Departement of mathematics, Technical University of Denmark, DK2800 Lyngby, Denmark, 1996.

[5] G. Buttazzo, G. Dal Maso «Shape optimization for Dirichlet problems : Relaxed formulation and optimality conditions », Appl. Math. Optim., n ${ }^{\circ}$ 23, 1991, 17-49.

[6] J. CÉA « Conception optimale ou identification de forme, calcul rapide de la dérivée directionnelle de la fonction coút », M.A.A.N., $\mathrm{n}^{\circ} 20$ (3), 1986, 371-402.

[7] J. CÉa, S. Garreau, Ph. Gulllaume, M. Masmoudi « The Shape and Topological Optimizations Connection », Comput. Methods Appl. Mech. Engrg., $\mathrm{n}^{\circ} 188$ (4), 2000, 713-726.

[8] R. DAUTRAY, J. LiONS « Analyse mathémathique et calcul numérique pour les sciences et les techniques », MASSON, collection CEA, 1987. 


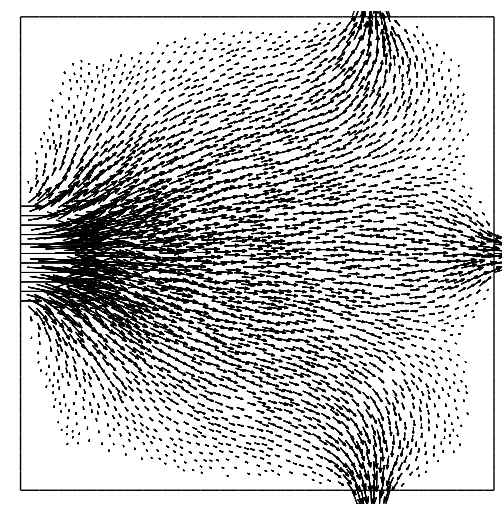

(a)

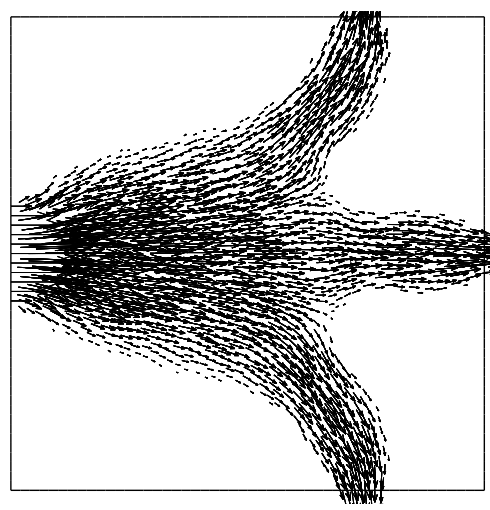

(c)

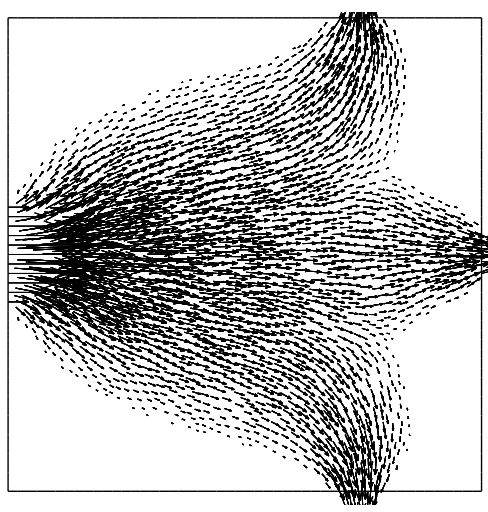

(b)

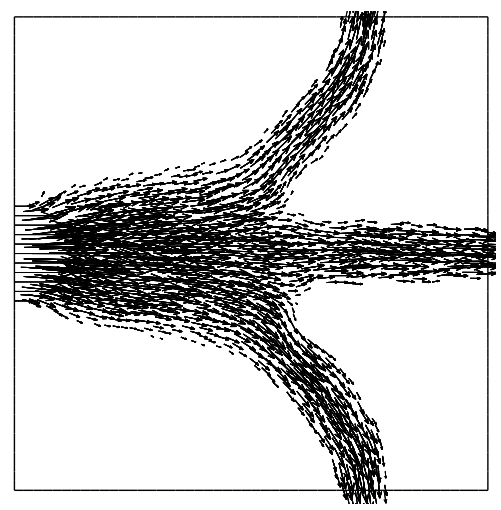

(d)

Figure 3. Velocity field obtained during optimization process.

[9] S. Garreau, Ph. Guillaume, M. Masmoudi « The topological asymptotic for pde systems : the elasticity case », SIAM J. Control Optim., n 39 (4), 2001, 1756-1778.

[10] R. Glowinski, O. Pironneau « Toward the computational of minimum drag profile in viscous laminar flow », Appl. Math. Model., $\mathrm{n}^{\circ}$ 1, 1976, 58-66.

[11] Ph.Guillaume, K.SID IdRIS « The topological asymptotic expansion for the Dirichlet Problem », SIAM J. Control. Optim., n 41(4), 2002, 1052-1072.

[12] Ph. Guillaume, K.SID IDRIS « Topological sensitivity and shape optimization for the Stokes equations », SIAM J. Control Optim., $\mathrm{n}^{\circ}$ 43(1), 2004, 1-31.

[13] M. Hassine, M. Masmoudi « The topological sensitivity analysis for the Quasi-Stokes problem », ESAIM, COCV J., vol. 10, 2004, 478-504.

[14] M. Masmoudi «The topological asymptotic », In Computational Methods for Control Applications, ed. H. Kawarada and J. Periaux, International Séries GAKUTO, 2002. 
229 - ARIMA - Volume 5 - 2006

[15] O. PironneAu « On optimal design in fluid mechanics », J. Fluid Mech., n ${ }^{\circ}$ 64, 1974, 97-110.

[16] J. Simon « Domain variation for Stokes flow », In Lecture Notes in Control and Inform. Sci., $\mathrm{n}^{\circ}$ 159, X. Li and J. Yang Eds. Springer, Berlin, 1990, 28-42.

[17] A. SCHUMACHER « Topologieoptimierung von bauteilstrukturen unter verwendung von lopchpositionierungkrieterien », thesis, Universitat-Gesamthochschule-Siegen, 1995.

[18] J. SokolowsKi, A. ZochowsKi « On the topological derivative in shape optimization », SIAM J. Control Optim., $\mathrm{n}^{\circ} 37$ (4), 1999, 1251-1272.

ARIMA - numéro spécial TAM TAM'05 